\title{
Disease activity in axial spondyloarthritis after discontinuation of TNF inhibitors therapy
}

\author{
Agata Sebastian ${ }^{1}$, Patryk Wojtala ${ }^{1}$, Łukasz Lubiński ${ }^{1}$, Małgorzata Mimier ${ }^{2}$, Arkadiusz Chlebicki ${ }^{1}$, \\ Piotr Wiland ${ }^{1}$
}

${ }^{1}$ Department of Rheumatology and Internal Medicine, Wroclaw Medical Hospital, Poland

${ }^{2}$ Department of Ophthalmology, Wroclaw Medical University, Poland

\begin{abstract}
Objective: Use of tumour necrosis factor inhibitors (TNFi) has proved to be an important step forward in the treatment of axial spondyloarthritis (axSpA), but the duration of the therapy as well as the management in case of low disease activity (LDA) or remission are not clearly established. Currently, the identification of potential predictors associated with the treatment discontinuation is the basic purpose of many clinical studies. The aim of this study was to analyze the influence of the discontinuation of TNFi therapy on the disease activity in patients with low disease activity.

Material and methods: The study included 65 patients; 47 of patients (72\%) were treated with etanercept, 16 (2\%) with adalimumab and 2 (3\%) with infliximab.

Results: The mean age of the patients was 45 years, the mean BASDAI score was 6.8 and VAS for low back pain was $76 \mathrm{~mm}$ at baseline. 54 patients with axSpA (83\%) achieved LDA after 9 months of anti-TNF therapy. During follow-up 40 patients (74\% of patients with LDA) had an increase of the disease activity after mean 14 weeks and needed to restart the treatment with TNFi. After restart of the therapy LDA was regained in all patients after mean 7 weeks. 11 patients (17\%) have never achieved LDA and 14 patients (22\%) had LDA longer than 6 months without relapse. At baseline higher levels of CRP and ESR were observed in patients with relapse of the disease at the end of treatment and with LDA shorter than 6 months.

Conclusions: Changes in the values of disease activity indicators (CRP, ESR) correlated with more stable response to TNFi therapy. Over $50 \%$ of patients who were treated with TNFi needed to restart the therapy. Treatment resumption allowed to regain a good clinical effect among affected patients.

Key words: ankylosing spondylitis, TNF inhibitors, low disease activity.
\end{abstract}

\section{Introduction}

The introduction of tumour necrosis factor inhibitors (TNFi) has been an important step forward in the treatment of chronic inflammatory diseases, including axial spondyloarthritis (axSpA). However, it should be noted that there is no formal proof that TNFi are in fact disease modifying in axSpA. It is not clear how long the therapy should last and which algorithm to choose when the disease activity is low or the remission occurs. Currently, the identification of potential predictors associated with

the treatment discontinuation is the basic purpose of many clinical studies. Based on the rules of therapeutic National Health Fund programme in Poland active ankylosing spondylitisis defined when BASDAI $\geq 4$ (Bath Ankylosing Spondylitis Disease Activity Index) and low disease activity (LDA) occurs when BASDAl is < 3 persist longer than 6 months. It is possible to reinstitute the therapy with TNF-inhibitor in any moment when BASDAI increases again more than 4. 
The aim of this study was to analyse the influence of the discontinuation of TNF-inhibitors therapy on the disease activity in patients with low disease activity (LDA).

\section{Material and methods}

We conducted a retrospective study of 65 patients (14 women and 51 men) with diagnosed axSpA, who were treated with TNFi in the Department of Rheumatology and Internal Diseases, Wroclaw University Hospital. The observation was done over the period 2009-2015. Patients were observed for an average of 9 to 48 months. Observations were based on monthly visits. The mean age of patients was 45 years (22-71). Before TNF-therapy all patients were treated for 6 months with two nonsteroidal anti-inflammatory drugs (NSAIDs), with no clinical effects. axSpA was diagnosed based on the Assessment of Spondyloarthritis International Society (ASAS) classification criteria for axial spondyloarthritis [1]. From the study group of patients 50 . had the radiographic signs of sacroiliitis (77\%, among them $75 \%$ were men) and 15 patients (23\%) had active sacroiliitis found in magnetic resonance imaging (MRI). MRI was done only in patients with no lesions in radiograms and who presented signs of axSpA. There was axial involvement observed in all patients while peripheral arthritis was confirmed in 13 patients (20\%), and 4 patients (40\%) with peripheral axSpA were treated with methotrexate and 5 (43\%) sulfasalazine. In almost all patients HLA-B27 was present and only 2 patients did not have this antigen. The extramusculosceletal indications to anti-TNF therapy were: uveitis - 14 patients (22\% of all patients), amyloidosis - 4 patients (6\%) and inflammatory bowel diseases 4 patients (6\%). In 47 cases (72\% of all patients) etanercept was used in the dose of $50 \mathrm{mg}$ s.c. per week, in 16 cases (25\%) adalimumab (40 mg s.c. every other week) and in 2 cases infliximab (5 mg/kg at 0, 14, 42 day and continuing every 8 weeks).

The response to therapy was defined as a reduction of $\geq 50 \%$ in BASDAI score or at least 2 units in VAS (visual analogue scale) for low back pain after 12 weeks of treatment with TNF inhibitor compared to the baseline visit.

TNFi was withdrawn after 6 months in patients who achieved LDA and it could be introduced again if the disease was active (BASDAI $\geq 4$ ) in accordance to the rules of therapeutic National Health Fund programme in Poland (group 1 - patients with relapse after LDA). Second group of patients had stabile LDA longer than 6 months after interruption of the TNF therapy (group 2 - patients with remission without relapse after LDA $\geq 6$ months). The last study group consisted of patients, who not achieved LDA for at least 6 months (group 3 - patients with no remission). During treatment patients were observed every month (group 1 and 3) and after achieving stabile LDA every 3 months (group 2).

We compared results of blood tests from patients at baseline and after treatment with TNFi in the last visit for each patient and these tests included erythrocyte sedimentation rate (ESR), C-reactive protein (CRP), white blood cells count (WBC), platelets count (PLT) and haemoglobin level $(\mathrm{Hb})$.

The statistical analysis was performed assuming the level of significance set up $0.05(p<0.05)$; the Student's T-test or analysis of variance - ANOVA were used. For the analysis we used the programme STATISTICA ver. 10.0.

\section{Results}

Among all 65 patients the mean BASDAI score was 6.8, mean VAS for low back pain $76 \mathrm{~mm}$ at baseline. Eleven patients (17\%) have never achieved LDA and 14 patients $(22 \%)$ had stabile LDA. Fifthy-four patients with axSpA (83\%) achieved LDA during the TNF-therapy. During follow-up 40 patients (74\% of patients with LDA) had increased disease activity after average 14 weeks and needed reapplication of TNFi after mean 7 weeks (2-13 weeks). The details are presented in Table I.

Fifthy-four patients with axSpA (83\%) the first BAS$\mathrm{DAI}<3$ points and improvement of minimum 2 units on

Table I. The mean values of blood tests, BASDAI and VAS score before introducing the TNF-inhibitors

\begin{tabular}{|c|c|c|c|c|c|c|c|c|c|}
\hline Group & $\begin{array}{c}\text { Number of } \\
\text { patients }\end{array}$ & $\begin{array}{c}\text { Age } \\
\text { (years) }\end{array}$ & $W B C \times 10^{9} / l$ & $\mathrm{Hb} g / \mathrm{dl}$ & $\mathrm{PLT} \times 10^{9} / \mathrm{I}$ & $\mathrm{ESR} \mathrm{mm} / \mathrm{h}$ & CRP $\mathrm{mg} / \mathrm{dl}$ & BASDAI & $\begin{array}{c}\text { VAS- low } \\
\text { back pain } \\
(0-100 \mathrm{~mm})\end{array}$ \\
\hline 1 & 40 & 41 & 7.6 & 13.2 & 326 & 32 & 24 & 6.1 & 75 \\
\hline 2 & 14 & 47 & 6.8 & 13.5 & 288 & 17 & 10 & 6.6 & 75 \\
\hline 3 & 11 & 45 & 7.2 & 14.0 & 287 & 22 & 16 & 7.6 & 79 \\
\hline p-ANOVA & & 0.27 & 0.43 & 0.28 & 0.27 & 0.08 & 0.18 & 0.009 & 0.68 \\
\hline
\end{tabular}

ESR - erythrocyte sedimentation rate; CRP - C-reactive protein; WBC - white blood cells count; PLT - platelets; Hb - haemoglobin level; BASDAI - Bath Ankylosing Spondylitis Disease Activity Index; VAS low back pain - visual analogue scale for low back pain; Group 1 - group of patients with relapse after LDA; Group 2-group of patients with remission without relapse after LDA $\geq 6$ months; Group 3 - group with no remission 
Table II. Mean CRP and ESR values at baseline and after treatment with TNFi

\begin{tabular}{|c|c|c|c|c|}
\hline Group & $\begin{array}{l}\text { CRP (mg/dl) } \\
\text { baseline }\end{array}$ & $\begin{array}{l}\text { CRP }(\mathrm{mg} / \mathrm{dl}) \\
\text { after treatment }\end{array}$ & $\begin{array}{l}\mathrm{ESR}(\mathrm{mm} / \mathrm{h}) \\
\text { baseline }\end{array}$ & $\begin{array}{c}\mathrm{ESR}(\mathrm{mm} / \mathrm{h}) \\
\text { after treatment }\end{array}$ \\
\hline 1 & 24 & 17 & 32 & 27 \\
\hline 2 & 10 & 3 & 17 & 11 \\
\hline 3 & 16 & 18 & 22 & 11 \\
\hline
\end{tabular}

ESR - erythrocyte sedimentation rate, CRP - C reactive protein, Group 1 - group of patients with relapse after LDA; Group 2 - group of patients with remission without relapse after LDA $\geq 6$ months; Group 3 -group with no remission, TNFi - tumour necrosis factor inhibitor

Table III. ASDAS score by groups

\begin{tabular}{|cccccccc|}
\hline Group & Number of patients & \multicolumn{3}{c}{ At baseline } & \multicolumn{4}{c|}{ At end of treatment } \\
\hline & $\begin{array}{c}\text { Average } \\
\text { ASDAS score }\end{array}$ & (Min.-Max) & SD & $\begin{array}{c}\text { Average } \\
\text { ASDAS score }\end{array}$ & (Min.-Max.) & SD \\
\hline 1 & 40 & 3.95 & $(1.9-5.4)$ & 0.73 & 1.91 & $(0.5-5.2)$ & 1.2 \\
\hline 2 & 14 & 3.57 & $(2.2-4.8)$ & 0.65 & 1.37 & $(0.6-2.7)$ & 0.56 \\
\hline 3 & 11 & 3.94 & $(2.9-48)$ & 0.63 & 1.76 & $(0.6-3.8)$ & 0.99 \\
\hline
\end{tabular}

SD - standard deviation

VAS low back pain, has been achieved after average 36 weeks of treatment.

At baseline increased CRP and ESR values were observed in group 1 versus group 2 and 3, but it was not statistically significant (respectively $p=0.18 ; 0.08$ ). In group 3 the average CRP value was $16 \mathrm{mg} / \mathrm{dl}$ at baseline and $18 \mathrm{mg} / \mathrm{dl}$ at the end of the observation. In patients without relapse after TNFi treatment (group 2) the average CRP was $10 \mathrm{mg} / \mathrm{dl}$ at baseline and normalized during treatment. The data was presented in Table II.

Based on the repeated measures ANOVA analysis it can be concluded that decrease in the ASDAS (The Ankylosing Spondylitis Disease Activity Score) value is significant for all groups considered $(p<0.05)$. However this descrease does not depend on the group ( $p=0.21$ ). It means that regardless of the group the decrease in ASDAS value is comparably significant. These results are visible on the Fig. 1 and in Table III.

Among patients who achieved stable LDA 11 patients (24\%) were treated with ETA (group 2), 2 (15\%) with ADA and 1 (50\%) with INF. LDA has never been achieved in $10(22 \%)$ of patients treated with ETA and $2(11 \%)$ with ADA.

In group 1 and 2 together 47 patients (87\%) have not taken the NSAIDs after mean 5 months of the treatment beginning. In 6 cases (11\%) the dose of NSAIDs was reduced to less than $50 \%$ of the initial dose.

At baseline no significant differences have been observed among men and women treated (Table IV). Stabile LDA was achieved by 11 men (21\%) and 3 women $(21 \%)$, respectively. LDA was not achieved by 9 men (18\%) and 2 women (14\%). The relapses were observed

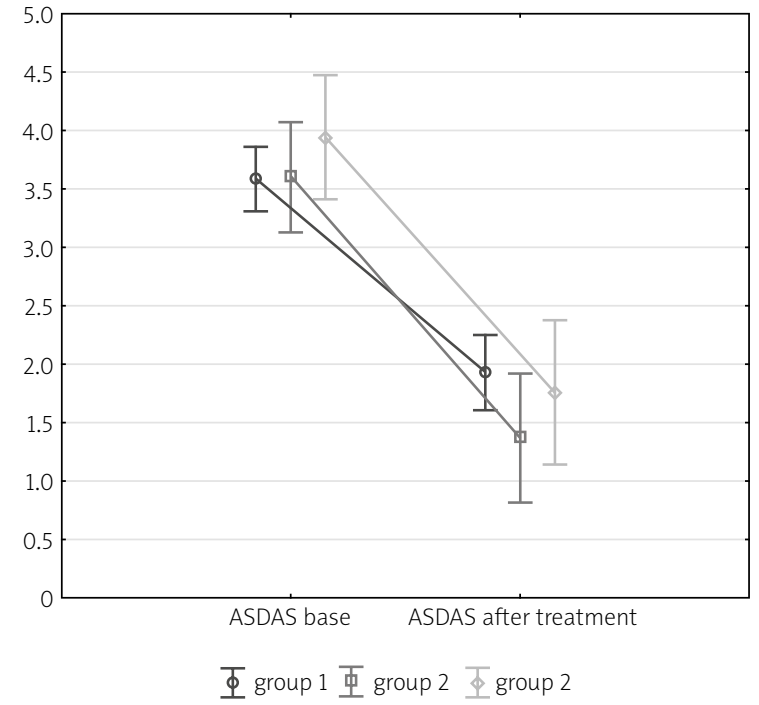

Fig. 1. Mean values of ASDAS score depending of time of measurement.

after mean of 15 weeks in men and after 11 weeks in women. In the analysis of laboratory results the $p$-value equals 0.4 and 0.7 for ESR and CRP respectively. The results suggest that there is no statistically significant difference between the analysed groups of patients, except $\mathrm{Hb}$ level, which was lower in women and VAS low back pain score was higher in women.

According to the concomitant clinical systemic involvement other than the axial spine and joints, the LDA was more frequent observed only in patients with uve- 
Table IV. Clinical characteristics of patients with asSpA according to the gender with mean values of laboratory findings

\begin{tabular}{|c|c|c|}
\hline & Men & Women $p-t$-Student \\
\hline Number of patients & 51 & 14 \\
\hline Age, mean (SD) years & 44 (SD 12) & 47 (SD 11) 0.82 \\
\hline Group without relapse & $11 / 51(21 \%)$ & $3 / 14(21 \%)$ \\
\hline \multicolumn{3}{|l|}{ Qualification parameters: } \\
\hline \multicolumn{3}{|l|}{ BASDAI } \\
\hline group with relapse & 6.0 & 6.50 .22 \\
\hline group without relapse & 6.4 & 7.8 \\
\hline \multicolumn{3}{|l|}{ VAS back pain } \\
\hline group with relapse & $72 \mathrm{~mm}$ & 86 mm 0.002 \\
\hline group without relapse & $73 \mathrm{~mm}$ & $82 \mathrm{~mm}$ \\
\hline \multicolumn{3}{|l|}{ ESR $(\mathrm{mm} / \mathrm{h})$} \\
\hline group with relapse & 29 & 410.4 \\
\hline group without relapse & 18 & 17 \\
\hline \multicolumn{3}{|l|}{$\operatorname{CRP}(\mathrm{mg} / \mathrm{l})$} \\
\hline group with relapse & 6 & 6.50 .76 \\
\hline group without relapse & 12.8 & 2.6 \\
\hline \multicolumn{3}{|l|}{$\mathrm{Hb}(\mathrm{g} / \mathrm{dl})$} \\
\hline group with relapse & 13.7 & 11.70 .007 \\
\hline group without relapse & 13.4 & 14.3 \\
\hline \multicolumn{3}{|l|}{ WBC count $\times 10^{9} / 1$} \\
\hline group with relapse & 7.9 & 6.80 .08 \\
\hline group without relapse & 7.2 & 5.6 \\
\hline \multicolumn{3}{|l|}{ PLT count $\times 10^{9} / 1$} \\
\hline group with relapse & 324 & 3330.94 \\
\hline group without relapse & 302 & 237 \\
\hline
\end{tabular}

ESR - erythrocyte sedimentation rate; CRP-C-reactive protein; WBC - white blood cells count; PLT - platelets; Hb-haemoglobin level; BASDAI - Bath Ankylosing Spondylitis Disease Activity Index; VAS low back pain - visual analogue scale for low back pain; Group 1 - group of patients with relapse after LDA; Group 2 - group of patients with remission without relapse after LDA $\geq 6$ months; Group 3 - group with no remission; $S D$ - standard deviation

itis by comparison with the group without LDA (79\% vs. $21 \%, p=0.6)$. Between patients with amyloidosis and inflammatory bowel disease 4 patients achieved LDA.

\section{Discussion}

The infiltrates such as histiocytes, macrophages, lymphocytes and plasmocytes are present in the sacroiliac joints during ankylosing spondylitisis at the very beginning. The early pathologic changes cause the formation of granulation tissue. Based on the immunohistochemical staging it was proved that in the case of active inflammatory changes the molecular expression was increased, including tumor necrosis factor (TNF) and transforming growth factor $\beta$ (TGF- $\beta$ ) $[2,3]$. The first step in the axSpA treatment consists of rehabilitation and pharmacological treatment.

Nonsteroidal anti-inflammatory drugs (NSAIDs) are recommended as the first line therapy in axSpA by the ASAS (The Assessment of SpondyloArthritis International Society). NSAIDs when used in maximal doses have positive impact on the course of the disease in patients with active axSpA, but they do not stop the structural changes in the long-term observation [4]. However, we have to acknowledge that the term bDMARDs is not completely correct as the disease-modifying aspect has not yet been proven in axSpA. There is an evidence supporting the efficacy of infliximab, etanercept, adalimumab, certolizum$\mathrm{ab}$ and golimumab in treatment of axSpA [5], which can be used in Poland in active axSpA - BASDAI $\geq 4$ and low 
back pain in VAS-scale $\geq 4 \mathrm{~cm}$, when NSAIDs are intolerant or ineffective. The response to therapy is defined as reduction of $\geq 50 \%$ in BASDAl score or at least 2 units in VAS compared to the baseline visit after 12 weeks of treatment with TNF inhibitor. In patients with axSpA LDA longer than 6 month causes discontinuation of the therapy. The resume of treatment is possible when the disease activity increases (BASDAI $\geq 4$ ).

The BASDAI-scale and the intensity of low back pain on VAS $[6,7]$ were currently the main measures for effectiveness of biological treatment and qualification criteria in axSpA. Although objective measures such as of CRP and ESR values are used, they are not basic tools in monitoring the biology treatment in Poland. Alongside BASDAl score, the disease activity score such as ASDAS or SASDAS, which include the ESR and CRP are recommended internationally to assess axSpA [8, 9].

However, we know that not in all cases with axSpA disease activity is correlated with increased CRP or ESR values. Actually, there is no widely used definition of remission and LDA that would include the normalization of inflammatory factors or changes in the radiological studies. BASDAI score and VAS low back pain are very subjective opinion of disease activity, made by patient himself. Until now, there has been no quantitative measure for objective monitoring axSpA activity such as biomarkers, which might affect the prediction of the duration of TNFi therapy.

The analysis revealed that ESR and CRP may be a prognostic factor. The values of these parameters were higher in patients who had relapses or had never reached the LDA than in patients who achieved remission irrespective of the TNFi. The value of CRP was associated with increased axSpA activity, similarly to other publications [10]. We observed the persistence of elevated CRP values through treatment in the group of patients who have not achieved LDA.

According to recent studies [11], serum CRP values correlate with clinical response in patients treated with TNFi. It was also useful for predicting of radiological signs of changes in sacroiliac joints recently. No disease-modifying medication has yet been able to stop the progression of the disease, as well as the backbone [12]. Normal CRP value failed to prevent radiographic progression. Molecules that block the activity of TNF-alpha are currently the standard therapy enabling effective treatment in axSpA.

In our study, a similar percentage of men and women have achieved long-term LDA and there was no recurrence of their symptoms after discontinuation of TNF inhibitor until the last visit. In the remaining patients, the mean time to relapse was 14 weeks, and was similar to the mean time described in other studies (from
14 to 24 weeks). The resulting percentage of LDA was also similar to the before published results of clinical trials around the world [13-15]. The inability to obtain of long-term LDA has led to periodic resume to the TNFi previously used. The continuing treatment [16] was required in most of the patients, who have been interrupted TNFi, in our study 48 patients (74\%). There was no difference observed between obtaining the LDA and used inhibitor of TNF and gender. At the beginning women had higher ESR, however, in our publication it has been not confirmed. The other authors reported previously, that the female gender might be a good prediction factor for the treatment of AS using TNFi. Other studies also observed that older age, longer duration of disease, severe inflammation of the sacroiliac joints, the occurrence of syndedmophytes at an early stage of the disease and elevated values of CRP were good markers of predicting the formation of the next syndesmophytes in women suffering from AS [17]. In the Outcome in AS International Study (OASIS) study, has been demonstrated that the correlation between increased activity AS (ASDAS scale) and the progression of radiological changes in the spine exist, particularly in men [18].

In our study patients who achieved LDA were older than those who did not have LDA and they had also lower ESR and serum CRP concentration at treatment beginning. At the time of qualification to the treatment they had similar levels of BASDAI and VAS of spinal pain compared to patients who did not end up treatment on a permanent basis. Taking into consideration additional indication to the treatment with a TNFi (like uveitis, amyloid disease, inflammatory bowel disease) LDA was observed more frequently by patients who suffered only from uveitis than by those who did not reach the LDA (79\% vs. 21\%). However, the sample size is too small to generalize these conclusions into the whole population so it requires further observation.

Therapy with TNFi is scientifically proven and currently the most effective therapy for patients suffered from axSpA. The use of biological treatment in AaxSpa is characterized by fast, good clinical response and the therapeutic effect persisted even for 3 years provided that the treatment is continued [14]. The safe use duration of TNFi in the treatment of axSpA has not been established yet. The longest clinical observation is eight years $[19,20]$. The inability to obtain the long-term stable form of LDA makes it necessary to re-use previously used TNF inhibitor therapy periodically but the benefits from the use of TNF inhibitors are still greater than the risk of potential complications.

In our study more than $50 \%$ of treated patients needed a re-use of TNFi. Therefore there is a need for further studies to determine whether and how long such treat- 
ment should be continued despite achieved LDA and whether the dose of the TNFi should be reduced to obtain long-term LDA [21, 22]. Due to the chronic nature of the axSpA all patients should also have possibility to return to the treatment with TNFi because it is the best therapy which is currently accessible.

\section{Conclusions}

Changes in the values of disease activity indicators (CRP, ESR) correlate with more stable response to TNFi therapy.

Over $50 \%$ of patients who were treated with TNFi needed to re-use the therapy. Treatment reinstitution was allowed regain a good clinical effect.

The authors declare no conflict of interest.

\section{References}

1. Akkoc N, Khan MA. ASAS classification criteria for axial spondyloarthritis: time to modify. Clin Rheumatol 2016; 35:14151423.

2. François RJ, Gardner DL, Degrave EJ, et al. Histopathologic evidence that sacroiliitis in ankylosing spondylitis is not merely enthesitis. Arthritis Rheum 2000; 43: 2011-2024.

3. Wu W, Ding Y, Chen Y, et al. Susceptibility to ankylosing spondylitis: evidence for the role of ERAP1, TGFb1 and TLR9 gene polymorphisms. Rheumatol Int 2012; 32: 2517-2521.

4. Braun J, van den Berg R, Baraliakos X, et al. 2010 update of the ASAS/EULAR recommendations for the management of ankylosing spondylitis. Ann Rheum Dis 2011; 70: 896-904.

5. van der Heijde D, Ramiro S, Landewe R, et al. 2016 update of the ASAS-EULAR management recommendations for axial spondyloarthritis. Ann Rheum Dis 2017; 76: 978-991.

6. Garrett S, Jenkinson T, Kennedy LG, et al. A new approach to defining disease status in ankylosing spondylitis: the Bath Ankylosing Spondylitis Disease Activity Index. J Rheumatol 1994; 21: 2286-2291.

7. Brandt J, Haibel H, Cornely D, et al. Successful treatment of active ankylosing spondylitis with the anti-TNF- $\alpha$ monoclonal antibody infliximab. Arthritis Rheum 2000; 43: 1346-1352.

8. Machado P, Navarro-Compán V, Landewé R, et al. How to calculate the ASDAS if the conventional CRP is below the limit of detection or if using high sensitivity CRP? An analysis in the DESIR cohort. Arthritis Rheum 2015; 67: 408-413.

9. Salaffi F, Ciapetti A, Carotti M, et al. Construct validity and responsiveness of the simplified version of Ankylosing Spondylitis Disease Activity Score (SASDAS) for the evaluation of disease activity in axial spondyloarthritis. Health Qual Life Outcomes 2014; 12: 129.

10. Arends $S$, Brouwer $E$, Efde $M$, et al. Long-term drug survival and clinical effectiveness of etanercept treatment in patients with ankylosing spondylitis in daily clinical practice. Clin Exp Rheumatol 2017; 35: 61-68.
11. Lee W, Reveille JD, Davis JC J, et al. Are there gender differences in severity of ankylosing spondylitis? Results from the PSOAS cohort. Ann Rheum Dis 2007; 66: 633-638.

12. Maas F, Arends S, Brouwer E, et al. Reduction in spinal radiographic progression in ankylosing spondylitis patients receiving prolonged treatment with TNF- $\alpha$ inhibitors. Arthritis Care Res 2016; doi: 10.1002/acr.23097.

13. Braun J, Deodhar A, Dijkmans B, et al. Efficacy and safety of infliximab in patients with ankylosing spondylitis over a twoyear period. Arthritis Rheum 2008; 59: 1270-1278.

14. Davis JC, Van Der Heijde D, Braun J, et al. Recombinant human tumor necrosis factor receptor (etanercept) for treating ankylosing spondylitis: a randomized, controlled trial. Enbrel Ankylosing Spondylitis Study Group. Arthritis Rheum 2003; 48: 3230-3236.

15.van der Heijde DM, Revicki DA, Gooch KL, et al. Physical function, disease activity, and health-related quality-of-life outcomes after 3 years of adalimumab treatment in patients with ankylosing spondylitis. ATLAS Study Group. Arthritis Res Ther 2009; 11: 124.

16. Braun J, Sieper J. Remission and possible discontinuation of biological therapy in axial spondyloarthritis. Clin Exp Rheumatol 2013; 31: 33-36.

17. Kang K, Kwok SK, Ju J, et al. The predictors of development of new syndesmophytes in female patients with ankylosing spondylitis. Scand J Rheumatol 2015; 44: 125-128.

18. Ramiro S, van der Heijde D, van Tubergen A, et al. Higher disease activity leads to more structural damage in the spine in ankylosing spondylitis: 12-year longitudinal data from the OASIS cohort. Ann Rheum Dis 2014; 73: 1455-1461.

19. Baraliakos X, Listing J, Fritz C, et al. Persistent clinical efficacy and safety of infliximab in ankylosing spondylitis after 8 years - early clinical response predicts long-term outcome. Rheumatology 2011; 50: 1690-1699.

20. Baraliakos X, Haibel H, Fritz C, et al. Long-term outcome of patients with active ankylosing spondylitis with etanercept-sustained efficacy and safety after seven years. Arthritis Res Ther 2013; 15: 67.

21. Song $\mathrm{IH}$, Haibel H, Poddubnyy D, et al. Withdrawal of biologic therapy in axial spondyloarthritis: the experience in early disease. Clin Exp Rheumatol 2013; 31: 37-42.

22. Pincus T, Braun J, Kavanaugh A, et al. Possible discontinuation of therapies in inflammatory rheumatic diseases - as with initiation of therapies, a shared decision between patient and rheumatologist. Clin Exp Rheumatol 2013; 31: 1-3. 